This article was downloaded by:[ANKOS 2006 ORDER Consortium]

On: 21 J anuary 2007

[ANKOS 2006 ORDER Consortium]

Access Details: [subscription number 758064771]

Publisher: Informa Healthcare

Informa Ltd Registered in England and Wales Registered Number: 1072954

Registered office: Mortimer House, 37-41 Mortimer Street, London W1T 3J H, UK

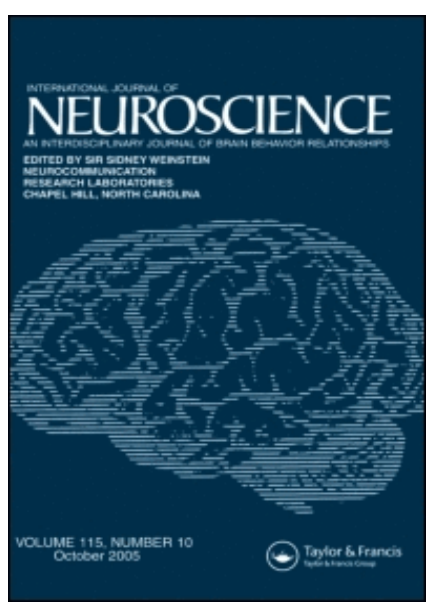

International J ournal of Neuroscience

Publication details, including instructions for authors and subscription information: http://www.informaworld.com/smpp/title content=t713644851

A WRIST-WALKER EXHIBITING NO "UNER TAN SYDNROME": A THEORY FOR POSSIBLE MECHANISMS OF HUMAN DEVOLUTION TOWARD THE ATAVISTIC WALKING PATTERNS Uner Tan ${ }^{\mathrm{a}}$

a Medical School, Department of Physiology, Cukurova University. Adana. Turkey

To link to this article: DOI: $10.1080 / 00207450600936866$

URL: http://dx.doi.org/10.1080/00207450600936866

Full terms and conditions of use: http://www.informaworld.com/terms-and-conditions-of-access.pdf

This article maybe used for research, teaching and private study purposes. Any substantial or systematic reproduction, re-distribution, re-selling, loan or sub-licensing, systematic supply or distribution in any form to anyone is expressly forbidden.

The publisher does not give any warranty express or implied or make any representation that the contents will be complete or accurate or up to date. The accuracy of any instructions, formulae and drug doses should be independently verified with primary sources. The publisher shall not be liable for any loss, actions, claims, proceedings, demand or costs or damages whatsoever or howsoever caused arising directly or indirectly in connection with or arising out of the use of this material.

(c) Taylor and Francis 2007 


\title{
A WRIST-WALKER EXHIBITING NO “UNER TAN SYDNROME": A THEORY FOR POSSIBLE MECHANISMS OF HUMAN DEVOLUTION TOWARD THE ATAVISTIC WALKING PATTERNS
}

\author{
ÜNER TAN \\ Cukurova University \\ Medical School, Department of Physiology \\ Adana, Turkey
}

\begin{abstract}
After discovering two families with handicapped children exhibiting the "Uner Tan syndrome," the author discovered a man exhibiting only wrist-walking with no primitive mental abilities including language. According to his mother, he had an infectious disease with high fever as a three months old baby; as a result, the left leg had been paralyzed after a penicilline injection. This paralysis most probably resulted from a viral disease, possibly poliomyelitis. He is now (2006) 36 years old; the left leg is flaccid and atrophic, with no tendon reflexes; however, sensation is normal. The boy never stood up on his feet while maturing. The father forced him to walk upright using physical devices and making due exercises, but the child always rejected standing upright and walking in erect posture; he always preferred wrist-walking; he expresses that wrist-walking is much more comfortable for him than upright-walking. He is very strong now, making daily body building exercises, and walking quite fast using a "three legs," although he cannot stand upright. Mental status, including the language and conscious experience, is quite normal. There was no intra-familiar marriage as in the two families mentioned earlier, and there is no wrist-walking in his family and relatives. There were no cerebellar signs and symptoms upon neurological examination. The brain-MRI was normal; there was no atrophy in cerebellum and vermis. It was concluded that there may be sporadic wrist-walkers exhibiting no "Uner Tan Syndrome." The results suggest that the cerebellum has nothing to do with human wrist-walking, which may rather be an atavistic trait appearing from time to time in normal individuals,
\end{abstract}

Received 17 May 2006.

Address correspondence to Prof. Dr. Üner Tan, Cukurova University, Medical School, Department of Physiology Adana 01330, Turkey. E-mail: unertan@cu.edu.tr; unertan37@yahoo.com 
indicating a live model for human reverse evolution. It was concluded that pure quadrupeds may sporadically appear due to random fluctuations in genotypes and/or environmental factors (hormonal or nutritional); the human development following the human evolution may be stopped in the stage of transition from quadrupedality to bipedality. That is, the activity of the philogenetically youngest supraspinal centers for bipedal walking responsible for suppression of the older supraspinal centers for quadrupedal gait may be interrupted at the atavistic level due to genetic and/or environmental factors. Consequently, it is assumed that these individuals prefer their natural wrist-walking to move around more quickly and efficiently.

Keywords brain, cerebellum, MRI, quadrupedal gait, Uner Tan syndrome, vermis, wrist-walking

\section{INTRODUCTION}

"Uner Tan Syndrome" is characterized by three main symptoms: quadrupedal wrist-walking, underdeveloped mental abilities, and primitive language (see Tan, 2005, 2006a). Contrary to the expectations of some experts (see Humphrey et al., 2005), the author has discovered a second family exhibiting "Uner Tan Syndrome," again the result of an intra-familiar marriage. Unlike in the first and second family described (see Tan, 2006b,c), there was no cerebellar hypoplasia or a mild vermal atrophy in the MRI scans (see Tan, 2006c) in the affected individual described earlier.

The pedigree analysis of the first and second families indicated an autosomal recessive transmission. Now the author has discovered another wrist-walking man, 36 years old, in another family with no intra-familiar marriage. The left leg of this person is paralyzed due to an infectious disease with high fever, probably poliomyelitis he had during his babyhood. After recovering from this disease, he never tried to stand up, preferred wrist-walking, refrained from using the paralyzed leg, and hopping on the right leg. Reflecting on the first and second families exhibiting the "Uner Tan Syndrome," the author expected a vermal atrophy in the cerebellum, hindering the upright posture and bipedal walk. Because the human poliovirus is known to be able to attach itsself to the human poliovirus receptor within the neural tissues including the cerebellar vermis among the other spino-cerebral structures, the possible polio-infection was expected as trigger for a vermal atrophy.

The case of this man will be presented in the present work in relation to the "Uner Tan Syndrome," that is, quadrupedal wrist-walking, mental retardation, primitive language, and vermal atrophy in MRI. 


\section{METHOD}

The man, now 36 years old, living in a village near Adana, suffered an illness with high fever when he was three months old. According to the father's and mother's reports, he could not move his left leg after a single penicillin injection during this illness, which most probably was poliomyelitis. As a baby he used bear-crawling without using his left leg. He did not want to stand uprighty during childhood. His father seriously and even rudely forced him to walk upright even supplying some walking devices, but the boy always refused. There is no other case exhibiting wrist-walking among the known relatives. As to the family tree, the family has seven children: the first is a 37 years old man (single); the second one is Mustafa, the wrist-walker, 36 years old, married with a new-born son; the third and fourth ones are females, 34 and 32 years old; the fifth one is a married woman with a daughter, five months old; the sixth one is a married man, having a son, six months old; and last one is a woman, single, 27 years old.

Nobody in the family tried bear-crawling during babyhood. They are all bipeds except Mustafa.

Upon neurological examination, there were no cerebellar signs and symptoms. The left leg was flaccid; no proprioceptive reflexes could be elicited; sensation was normal.

Mustafa is a shoe-shiner working in the American Airbase, Incirlik, Adana. $\mathrm{He}$ is quite normal in language and other intellectual activities, with his conscious experience also being in excellent state. He attended the primary school for only three years. Mini-mental state examination test (see Tan, 2006a) standardized for uneducated people showed a full intellectual capacity.

T1-weighted cerebral MRI scans were made, to detect abnormalities in cerebral and cerebellar structures, especially the basal ganglia, cortex, cerebellum, and cerebellar vermis. The examinations were performed with a 1.5 $\mathrm{T}$ magnet system, and included both the sagittal and axial $0.5 \mathrm{~mm}$ sequences.

\section{RESULTS}

\section{Tripedal Gait}

Although the man uses a wheel-chair to go to work, he walks rather fast tripedally if necessary. For instance, he went through the steps of the hospital nearly running upstairs and downstairs. Actually, he is a strong man, visiting the training center in the American Air-Base in Incirlik. He is a typical wrist-walker, using two hands and one foot. He cannot use the left leg because of its paralysis. 


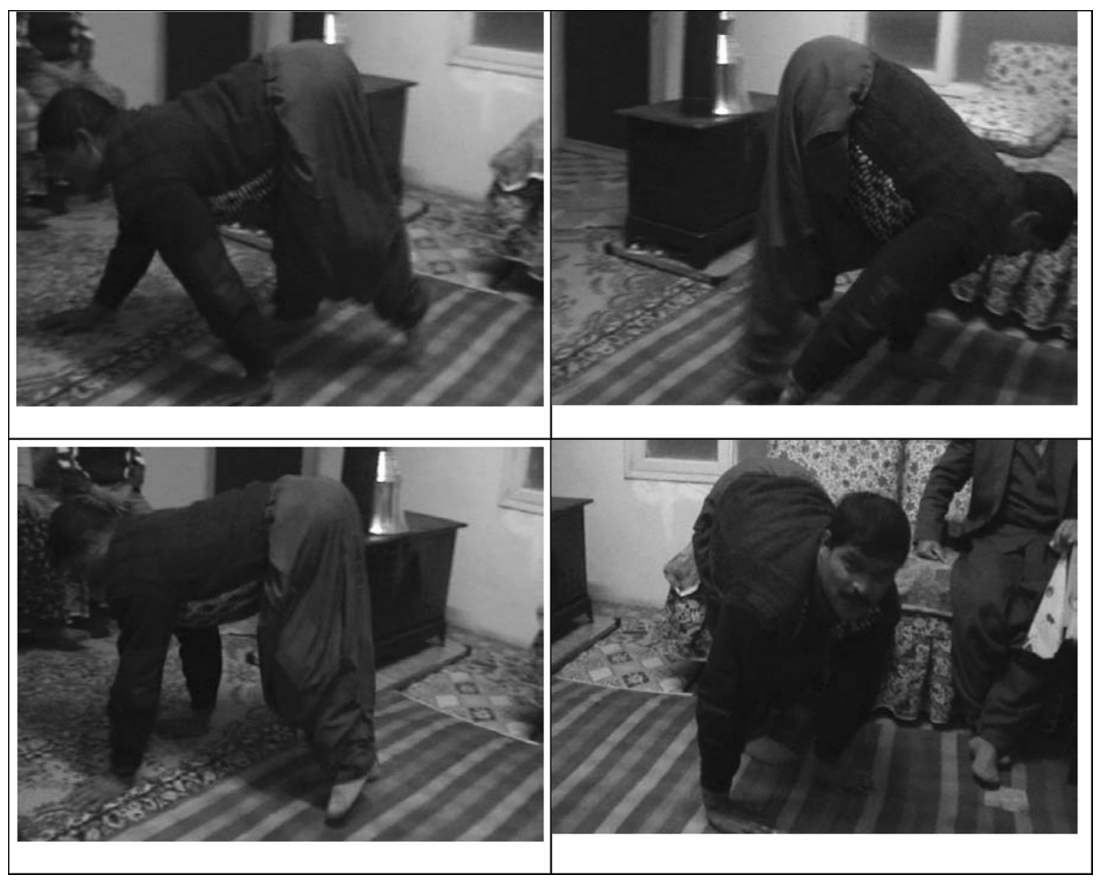

Figure 1. Walking patterns of a wrist-walking man. The left leg is paralyzed following possible poliomyelitis. (See Color Plate XVIII at end of issue.)

He easily and speedily runs up and down the steps of the house. He cannot stand upright without help, although he sits upright without bending the head or the body. He always rejected the physiotherapy, despite the father's rather rude demands, and preferred the wrist-walking because he always claimed that it is much easier for him to walk on both hands and the intact leg, tripedally; he has disliked the walking aids for an upright posture since childhood. During walking, the hands and arms are used to walk, whereas the single normal leg is hopping (tripedal gait). Mustafa's walking patterns are illustrated in Figure 1.

\section{MRI Scans}

The sagittal and transvers brain MRI scans are shown in Figure 2. There were no abnormalities in the brain structures including cerebral cortex, brain ventricles, basal ganglia, corpus callosum, cerebellum, and cerebellar vermis. 

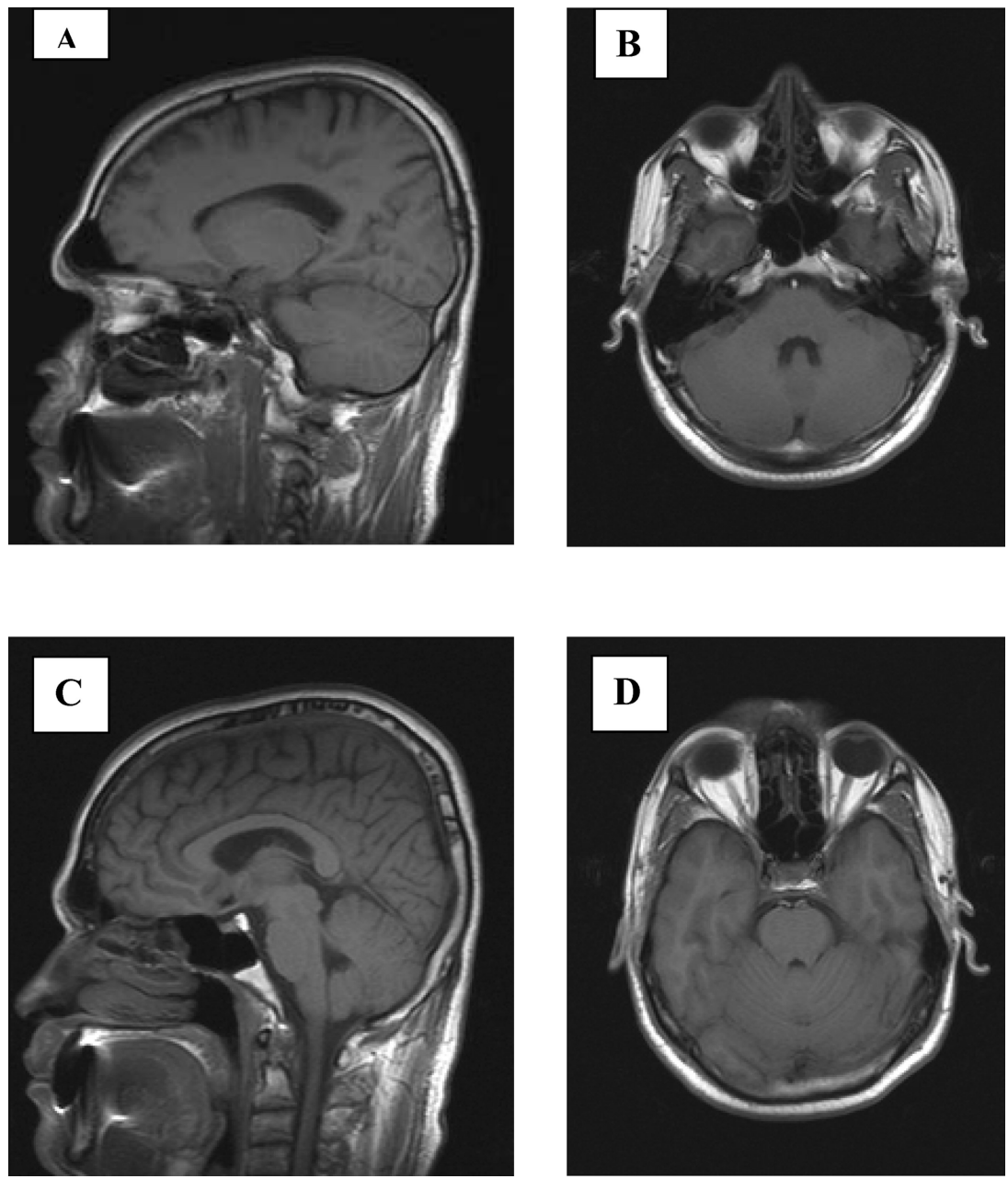

Figure 2. MRI scans from Mustafa's brain. A: sagittal cerebellum; C: sagittal vermis; B: transverse cerebellum; D: transverse vermis.

\section{Mental Abilities}

Mustafa was in Primary School for only three years. Then he stopped going, because he did not like the school. In spite of this, his language is well developed, his speech is fluent, using words necessary for everyday use. He gave correct answers to the questions in the mini-mental state examination test. So, his mental capacities including the speech and conscious experience were 
rather normal. Actually, he seemed to be a rather bright person when the author talked to him.

\section{DISCUSSION}

The present work has described a wrist-walker (Mustafa) with normal brain structures including cortex, basal ganglia, corpus callosum, cerebellum, and cerebellar vermis. He uses two hands (flat on the ground) and one foot during walking because the left leg has been paralyzed as a result of a possible poliomyelitis he suffered when he was three months old. He has never tried to stand up during childhood. He has always rejected to use walking aids for upright walking despite his father's rather rude instructions. Interestingly enough, he preferred wrist-walking, because it enabled him to walk around fast and quite efficiently.

The MRI results were not consistent with the hypothesis of the present work. Namely, it was expected that Mustafa's wrist-walking may be due to a vermal atrophy as the result of the suspected poliomyelitis because there were vermal atrophies in the wrist-walkers of the first and second families discovered (see Tan, 2005; Tan, 2006a,b,c). It is known that the spinal motor neurons may be partially destroyed by the poliomyelitis virus; other sites within the brain such as s medulla, cerebellar vermis, midbrain, thalamus, and cerebral cortex may also be affected as well (Bodian, 1949; Baker \& Cornwell, 1954; Curnen \& Chamberlin, 1962). The MRI scans showed, however, that all of the cerebral structures seemed to be normal. Therefore, Mustafa's wrist-walking cannot be attributed to a vermal atrophy. That might indicate that wrist-walking may occur without a vermal hypoplasia. In comparison, there were the same vermal atrophies in the ataxic-biped and quadruped individuals of the first and second families described in another work of mine (see Tan, 2005; Tan, 2006a,b,c).

In this case, the author assumed to be confronted with a psychological variable, that is, the conscious choice between upright bipedal walking and quadrupedal wrist-walking. There seems to be no genetic defect in the presented case because there was no intra-familiar marriage, and no wrist-walking appeared in other relatives. Mustafa preferred wrist-walking since childhood and all of his adult life. Mustafa used bear-crawling during his babyhood; he did not stand upright during maturation. Bear-crawling has been observed in some babies before standing up and starting to walk on two legs. Children walking on all fours were excellently described by Hrdlicka as early as 1928 (see Hrdlicka, 1928). This author has also reported that "in the two preceeding 
numbers of this Journal there were reported eleven, or, more strictly, ten cases of human children, before walking, ran for a shorter or longer time on all fours." These were healthy, normal children, generally beginning to crawl on all fours at approximately 10 months of age, lasting for about 8 months. So, some babies prefer bear-crawling (wrist-walking) and then stand up and start to walk bipedally. However, in some cases they may continue preferring the wrist-walking for the rest of their lives. This is, however, fairly seldom, and seen all over the world from time to time, according to e-mails sent to me from different parts of Turkey and other countries. These children are born strong and healthy, have ample vitality, and frequently remain above the average in strength, activity, and even mentality (see also Hrdlicka, 1928). A man in the United States has reported about the Tennessee Titans that "I was up there for football camp and on the way back from a practice, we saw them running down the hill. It was insane ... they are supposed to go up and down the hill five times in five minutes (not sure if it was multiple sets). While we were walking by, there were three or four guys basically bear crawling their way up." A friend of mine has seen a man hurrying across the street on all fours, then disappeared quickly. Thus, some normal people prefer the wrist-walking during adulthood.

These examples suggest that wrist-walking may be an inherent property of some human beings, which may reflect a reverse evolution toward the quadrupedal walk in human ancestors. This atavistic walking pattern may be inhibited by the supraspinal structures evolved later along with a habitual upright posture, resulting in bipedal gait. There are benefits and handicaps of the qaudrupedal gait. As seen in the quadruped children and adults, the wrist-walkers can run very fast. This is however disadvantageous for the hand skill to be able to perform actions that normally need a well-developed hand skill. On the other hand, the bipedal gait is not fast, while the freed hands may be used for skilled manual activities. The benefits of the quadrupedal gait may be seen in animals, especially during hunting and escaping from hunters.

The sporadic wrist-walkers presented herein apparently do not exhibit the "Uner Tan Syndrome," which is a combination of three main symptoms: quadrupedal wrist-gait, primitive intelligence, and primitive language. There is a vermal atrophy in the "Uner Tan Syndrome," whereas the normal wrist-walkers with normal mental abilities do not show a vermal atrophy. The quadrupedal gait is seen in some children from time to time, but they stand up and start to walk on two feet, using a bipedal gait for the rest of their life. On the other hand, there are adult wrist-walkers with normal cognitive abilities including the language. During normal development of the children, similar to the evolution of human locomotion, there are some stages consisting of 
creeping, normal crawling using the knees or in a lesser extent bear-crawling using the hands and feet, and finally the upright standing with bipedal gait. Apparently, some children stop the locomotive development at the stage of bear-crawling, preferring the wrist-walking for the rest of their lives. So, the normal development of locomotion may be interrupted during transition from quadrupedality to bipedality, and stop the further development to the final human erect posture and bipedal gait. This punctuated development may be considered a backward step toward the atavistic locomotion, suggesting a reverse evolution or devolution, which means the reacquisition of the same character states as those of ancestor populations by derived populations (Teotonio \& Rose, 2001).

The reverse evolution may be genetic and/or environmental in origin. The randomly occurred small genetic knockouts or the lost of genes recently acquired through evolution may be responsible for these randomly occurred wrist-walkers within the biped human populations. This may cause an interruption in the transition from a human atavistic locomotion seen during babyhood to a bipedal locomotion seen in adult humans. Accordingly, there is evidence in the scientific literature, suggesting that the hypothesis of reverse evolution is plausible and testable (see e.g., Teotonio \& Rose, 2000, 2001, 2002; Porter \& Crandall, 2003). Environmental factors may depend on the nutritional and hormonal status of the intrauterine environment during fetal growth. The genetic and/or environmental factors may then result in the absence of the supra-spinal structures responsible for the development of the upright standing and bipedal walking, and the baby may remain in the stage of transition from quadrupedality to bipedality. This may in turn appear as a reverse evolution in human locomotion. That is, in this case, the supraspinal structures suppressing the atavistic cerebro-spinal mechanisms responsible for the quadrupedal wrist-walking may fail in their neural efficiency to inhibit the ancestral brain centers for atavistic walking patterns. It can thus be concluded that the quadrupedal wrist-walking may not necessarily be the result of a brain damage; it may be just not a normal human brain genetically and/or environmentally programmed for a quadrupedal wrist-gait, and stopped at the stage of transition from quadrupedality to bipedality.

\section{CONCLUSIONS}

A 36-year-old man preferring the wrist gait since his babyhood was presented in this work. Despite his wrist-walking, he has no "Uner Tan Syndrome," because two further characteristics of this new syndrome, the primitive mental 
abilities, and the primitive language, were absent in this rather bright person. There was no quadrupedality among his relatives. There were no abnormalities in brain MRI scans. Except his left leg paralyzed probably as a result of poliomyelitis he had during babyhood, there were no pathological signs and symptoms upon neurological examination. There are further sporadic cases exhibiting qaudrupedal wrist-walking in Turkey and in some other countries. This can be attributed to a devolution toward human ancestors in relation to the quadrupedal gait. It was suggested that there may be an interruption in the transition from quadrupedality to bipedality during a child's development, that is during the development of philogenetically younger supraspinal centers suppressing the brain mechanisms responsible for the atavistic qaudrupedal gait. Genetic and/or environmental factors may stop the normal transition from qaudrupedality to bipedality. As a result of this devolutionary process, such an individual may not further develop into Homo erectus exhibiting the upright posture and bipedal gait, which is the most prominent feature for the uniqueness of human beings.

\section{REFERENCES}

Baker, A. B., \& Cornwell, S. (1954). Poliomyelitis. X. The cerebellum. AMA Archive of Neurology and Psychiatry, 71, 455-465.

Bodian, D. (1949). Histopathologic basis of clinical findings in poliomyelitis. The American Journal of Medicine, 6, 563-578.

Curnen, E. C., \& Chamberlin, H. R. (1962). Acute cerebellar ataxia associated with poliovirus infection. The Yale Journal of Biology and Medicine, 34, 219-233.

Hrdlicka, A. (1928). Children running on all fours. American Journal of Physical Anthropology, 11, 149-185.

Humphrey, N., Skoyles, J. R., \& Keynes, R. (2005). Human hand-walkers: Five siblings who Never stood up (online). London: LSE Research Online.

Porter, M. L., \& Crandall, K. A. (2003). Lost along the way: The significance of evolution in reverse. TRENDS in Ecology and Evolution, 18, 541-547.

Tan, U. (2005). Unertan Syndrome; qaudrupedality, primitive language, and severe mental retardation; a new theory on the evolution of human mind. NeuroQuantology, $4,250-255$.

Tan, U. (2006a). A new syndrome with quadrupedal gait, primitive speech, and severe mental retardation as a live model for human evolution. International Journal of Neuroscience, 116, 361-369.

Tan, U. (2006b). Evidence for "UnerTan Syndrome" and the evolution of human mind. International Journal of Neuroscience, in press.

Tan, U. (2006c). Evidence for UnerTan syndrome as a human model for reverse evolution. International Journal of Neuroscience, in press. 
Teotonio, H., \& Rose, M. R. (2000). Variation in the reversibility of evolution. Nature, 408, 463-466.

Teotonio, H., \& Rose, M. R. (2001). Perspective: Reverse evolution. Evolution, 55, 653-660.

Teotonio, H., \& Rose, M. R. (2002). Reverse evolution of fitness in Drosophila melanogaster. Journal of Evolutionary Biology, 15, 608-617.

Turkmen, S., Demirhan, O., Hoffmann, K., Diers, A., Zimmer, C., Sperling, K., \& Mundlos, S. (2006). Journal of Medical Genetics, 43, 461-464. 